\title{
Volverse a YHWH, el Dios de la justicia: estudio de Amós 4*
}

\author{
Pinky Riva**
}

Recibido: 24 de Abril de 2014 • Aprobado: 28 de Junio de 2014

\section{Resumen}

La metáfora de las vacas de Basan que se menciona en el cuarto capítulo de Amós ha suscitado muchas interpretaciones (como, por ejemplo, que las mujeres del reino norte de Israel eran muy gordas o infieles a sus maridos). La autora toma como punto de referencia para la interpretación de esta figura la revolución agrícola que se dio en Israel por la introducción del uso del ganado bovino para la agricultura y el consecuente surgimiento de la monarquía para la protección del excedente económico producido de esta manera, pero no repartido como anteriormente se hacía con la tierra según la experiencia liberadora del Éxodo. Este cambio productivo impulsa la transición de la sociedad tribal a la monarquía que da pie a la fuerte crítica de Amós contra las injusticias sociales.

Palabras clave: injusticia social, mujer, culto, justicia, bondad

* El texto es producto de la labor investigativa de la autora

** Pinky Riva es laica, católica, biblista, realizó sus estudios de Biblia en la Universidad Bíblica Latinoamericana (UBL) de San José, Costa Rica. Coordinadora del Servicio Bíblico Permanente (SEBIP), parte de la Red Ecuménica de Teólogas de La Paz- Bolivia. Correo electrónico: labiblista@sebip.org 


\title{
Turning to YHWH, the God of justice: study of Amos 4
}

\begin{abstract}
The metaphor of the cows of Bashan mentioned in the fourth chapter of Amos has aroused many interpretations (as, for instance, that women in the north kingdom of Israel were very fat or unfaithful to their husbands). The author takes as point of reference for the interpretation of this figure the agricultural revolution that occurred in Israel by the introduction of the use of cattle for agriculture and the resulting rising of the monarchy to protect the economic surplus produced this way, but not distributed as was previously done with the land according to the liberating experience of the Exodus. This productive change drives the transition from tribal society to monarchy which gives rise to the strong criticism of Amos against social injustices.
\end{abstract}

Keywords: social injustice, women, worship, justice, goodness

\section{Revenir a YHWH, le Dieu de la Justice : étude d’ Amos 4}

\section{Résumé}

La métaphore des vaches du Basan qui est mentionné dans le chapitre 4 d'Amos a suscité beaucoup d'interprétations (comme, par exemple, que les femmes du royaume nord d'Israël étaient très grosses et infidèles à leurs maris). L'auteur prend comme point de référence pour l'interprétation de cette figure, la révolution agricole qui a eu lieu en Israël par l'introduction des bovins dans l'agriculture et l'apparition conséquente de la monarchie pour la protection de l'excédent économique produit de cette façon, mais non distribué comme cela l'était antérieurement avec la terre, selon l'expérience libératrice de l'exode. Ce changement productif fait avancer la transition de la société tribale à la monarchie qui donne lieu à la forte critique d'Amos contre les injustices sociales.

Mots Clés: injustice sociale, femme, culte, justice, bonté

\section{Contexto General}

Amós no fue un profeta 'profesional', vinculado a institución alguna (Am 7, 1415; Biblia de Jerusalén). Se encontraba comprometido con la sufrida vida de los excluidos en Israel y con YHWH, quienes constituían su fuerza e impulso (Am $3,78 ; 7,15)$. Su nombre significa "YHWH carga o sustenta". Fue un portavoz de 
Dios y del pueblo. Escuchó en el grito de los pobres su búsqueda de YHWH. Trabajaba donde encontrara trabajo: a veces, lo vemos cuidando ovejas (Am 1,1); otras, ganado; otras, está cultivando sicomoros (Am 7,14). El tiempo de su ministerio debió haber durado unos dos años, alrededor de 760 a. C., durante el reinado de Jeroboán II, cuando fue expulsado del Santuario de Betel por orden del rey (Am 7, 10-13). Amós era natural de Técoa, a $18 \mathrm{~km}$ al sur de Jerusalén, en la sierra de Judá. Era, por tanto, del sur (Am 1,1). Pero su predicación fue en el Reino de Israel: se ubicó en Betel (Am 7, 10-17) y Samaria (Am 3, 9; 4,1), es decir en el Norte.

\section{División del libro de Amós}

Podemos dividir el libro de Amós de la siguiente forma (Asurmendi, 2000):

- Título: 1,1-2.

- Oráculos contra Judá, Israel y las naciones vecinas: 1, 3-2, 16.

- Oráculos contra Israel: 3, 1-6,14

- Cinco visiones: 7, 1-9, 10

- Oráculos de salvación: 9, 11-15.

\section{Claves genérales de lectura}

Según el análisis que Amós hace de la vida en su libro, proponemos las siguientes claves, presentadas para una mayor facilidad como preguntas a responder en el texto:

- ¿Cuál es la injusticia que se comete en Israel?

- ¿Quiénes son los responsables de las injusticias?

- ¿Quiénes son las víctimas que sufren la injusticia?

- ¿Cómo actúa Dios en cada situación a través del profeta?

- ¿Qué solución propone el profeta?

Amós es parte del movimiento de resistencia a la monarquía israelí. Esta se había instalado firmemente en el sur y creó una sociedad desigual entre la corte asentada en la ciudad y el pueblo campesino. El proyecto de la monarquía va en contra de la vivencia del Éxodo, en la que diferentes grupos oprimidos se ponen en camino siguiendo al Dios liberador y luchando por una sociedad justa e incluyente para todo el pueblo. Amós apuesta por ese proyecto de sociedad (tribal) para el pueblo. Anuncia "desgracias" para aquellos grupos causantes del sufrimiento y la miseria del pueblo. 


\section{El texto}

En Amós 4, 1-13 se advierte:

Nuestro texto está dentro de los oráculos a Israel en el Reino del Norte.

${ }^{1}$ Escuchad esta palabra, vacas de Basán, que estáis en la montaña de Samaria, que oprimís a los débiles, que maltratáis a los pobres, que decís a vuestros maridos: ‘¡Traed, y bebamos!'

${ }^{2}$ El Señor Yahveh ha jurado pro su santidad: He aquí que vienen días sobre vosotras en que se os izará con ganchos, $\mathrm{y}$, hasta las últimas, con anzuelos de pescar.

${ }^{3}$ Por brechas saldréis cada una a derecho, y seréis arrojadas al Hermón, oráculo de Yahveh.

${ }^{4}$ ¡Id a Betel a rebelaros, multiplicad en Guilgal vuestras rebeldías, llevad de mañana vuestros sacrificios cada tres días vuestros diezmos; ${ }^{5}$ quemad levadura en acción de gracias, y pregonad las ofrendas voluntarias, voceadlas, ya que es eso lo que os gusta, hijos de Israel!, oráculo del Señor Yahveh.

${ }^{6}$ Yo también os he dado dientes limpios en todas vuestras ciudades, y falta de pan en todos vuestros lugares; ¡y no habéis vuelto a mí! oráculo de Yahveh.

7 También os he cerrado la lluvia, a tres meses todavía de la siega; he hecho llover sobre una ciudad, y sobre otra ciudad no he hecho llover; una parcela recibía lluvia, y otra parcela, falta de lluvia, se secaba; ${ }^{8}$ dos, tres ciudades acudían a otra ciudad a beber agua, pero no calmaban su sed; iy no habéis vuelto a mí!, oráculo de Yahveh.

${ }^{9}$ Os he herido con tizón y añublo, he secado vuestras huertas y viñedos; vuestras higueras y olivares los ha devorado la langosta; iy no habéis vuelto a mí!, oráculo de Yahveh.

${ }^{10} \mathrm{He}$ enviado contra vosotros peste, como la peste de Egipto, he matado a espada a vuestros jóvenes, mientras vuestros caballos eran 
capturados; he hecho subir a vuestras narices el hedor de vuestros campamentos, iy no habéis vuelto a mí!, oráculo de Yahveh.

${ }^{11}$ Os he trastornado como Dios trastornó a Sodoma y Gomorra, habéis quedado como un tizón salvado de un incendio; iy no habéis vuelto a mí!, oráculo de Yahveh.

${ }^{12}$ Por eso, así voy a hacer contigo, Israel, y porque esto voy a hacerte, prepárate, Israel, a afrontar a tu Dios. ${ }^{13}$ Porque está aquí quien forma los montes y crea el viento, quien descubre al hombre cuál es su pensamiento, quien hace aurora las tinieblas, y avanza por las alturas de la tierra: Yahveh, Dios Sebaot es su nombre.

\section{División del texto}

Podemos dividir el texto en cuatro partes. Es una serie de oráculos:

1. Por las mujeres a los opresores (vv.1-3).

2. Por el culto a la injusticia (vv. 4-5).

3. Cinco oráculos de escarmiento (vv. 4-11).

4. Sentencia final (vv. 12-13).

\subsection{Por las mujeres a los opresores v.1-3}

Para entender la metáfora de las vacas de Basán es importante analizar el papel del buey en la sociedad en el tiempo de la monarquía.

La revolución agrícola, impulsada por la monarquía, y, con ella, la presencia de los bueyes constituyen elementos de suma importancia. La introducción del arado con lámina de hierro jalado por el buey aumentó significativamente la producción agrícola, pero el enriquecimiento no fue igual para todas las personas del pueblo de Israel (1S 13, 19-21): aparecieron las desigualdades sociales. Quien no tenía las mejores tierras estaba excluido de este avance tecnológico y quedaba cada vez más atrás económicamente. Se iban acentuando las diferencias de ingreso entre familias, clanes y tribus.

"Saúl, que venía entonces del campo detrás de los bueyes [...]" $(1 \mathrm{~S} 11,5)$. Este capítulo se refiere dos veces a los bueyes (1S 11,7). En todo el Primer Libro de Samuel, la palabra buey aparece 27 veces. En el Código de la Alianza (Ex 20, 
22-23,19), texto que se considera perteneciente a esa época, aparece 25 veces. Específicamente, podemos ver la importancia de la palabra en Éxodo 22, 28-30. Incluso se formuló un proverbio reconociendo que la fuerza del buey aumenta la producción y genera acumulación $(\operatorname{Pr} 14,4)$.

Es posible que nunca hayamos escuchado hablar de la presencia del buey en la época de transición entre la experiencia tribal y la monarquía. Podemos percibir su importancia, comparable con la introducción de la mecanización y el empleo de tractores y cosechadoras en la agricultura finales del siglo XIX. Este elemento constituyó una revolución tecnológica en la agricultura.

El alimento del buey se encontraba en las tierras antes ocupadas para producir alimentos. En consecuencia, aumentó el número de las personas sin tierra. Un proverbio de los campesinos sin tierra de hoy en Brasil reza, por ejemplo: "donde el buey berra el pueblo está sin tierra". Pero no solo ocurría eso. Aumentó también el hambre de la población. Otro dicho popular refleja bien esa realidad: "donde el buey come, el pueblo pasa hambre". Consecuentemente, aumentaron también los conflictos, la lucha por el liderazgo y el poder.

Podemos encontrar varios textos que testimonian el aumento de la desigualdad socioeconómica entre la población a finales del período tribal alrededor del 1200 a. C:

- El propio Saúl pertenecía a una familia acomodada (1S 9, 1-9) que tenía siervos (v.5) y bueyes (1S 11,5).

- Como en la época de la formación de Israel, las personas marginadas, los hapirus o hebreos, ya se organizaban nuevamente en bandas de mercenarios (1S 14,21; 22,2; 27, 8-12).

- La sociedad israelita, a final del tiempo de los jueces, ya estaba dividida en clases (1S 25). La solidaridad que había unido al pueblo en el Éxodo, poco a poco, cedió a los intereses de los grupos más poderosos.

Resultaba necesario proteger el ganado. El buey constituía uno de sus bienes más valiosos. También se hacía imperativo garantizar la libre circulación de las mercancías de los campesinos más ricos. Surge entonces la necesidad de un ejército permanente. Hasta podríamos decir que la posesión de bueyes exige un ejército. Podemos leer en el texto que Saúl convocó a los dueños de los bueyes y los amenazó con la pérdida de su ganado (1S 11,7). Fue un motivo más para tener un rey. No por azar el primer rey surgió próximo al entronque de las rutas comerciales Norte-Sur y Este-Oeste, en los alrededores de Jerusalén.

La causa principal que llevó a imponer la monarquía en Israel fue la necesidad de garantizar los intereses económicos, políticos e ideológicos de los más acomodados contra la resistencia popular y las amenazas externas. Esta, sin lugar 
a dudas, es la experiencia vivida por Amós, quién como vimos era un simple ganadero y un granjero. Cuando quiso decírselo claramente a las mujeres indulgentes de Israel, las llamó vacas gordas. El ganado de Basán - en el norte de Israel, hoy en día ולוגה תמר (Ramat ha-Golan), los Altos de Golan¹- era conocido por ser gordo y sano.

En el texto bíblico, encontramos algunas referencias más a este tema. Por ejemplo, en Salmos 22,13 se mencionan los fuertes toros de Basán; Ezequiel 39,18 habla del ganado engordado en Basán. Nos equivocamos si pensamos que tiene que ver con el ideal de una mujer hermosa muy delgada, tema que es algo moderno. Especialmente en los tiempos de antigüedad, ser carnudo era una muestra de 'riqueza'. Sin embargo, podemos dar por sentado que en ningún punto de la historia humana, a ninguna mujer le ha gustado que se le llamara vaca gorda.

La intención del profeta de usar la metáfora de vacas de Basán es llamar la atención sobre el lujo que disfrutaban las mujeres ricas y sobre cierta voluptuosidad y sensualidad que su extravagante estilo de vida les dio la oportunidad disfrutar. Amós aquí hace referencia a mujeres y varones malos, opulentos, perezosos, bajo la idea de novillos engordados, que pronto estaban por salir a ser sacrificados. Es posible que estas mujeres no hayan sido las directamente responsables por el maltratamiento de los pobres. Pero sus demandas insensatas de lujos hicieron que sus esposos hicieran injusticias aún más grandes. Amós denuncia los pecados de Israel y el castigo venidero de Dios. Parece que se dirige a las esposas de los ricos de Samaria, a quienes llama vacas de Basán, aunque algunos autores piensan que está referido a los nobles por el hecho de que se refiere a "vuestros señores". El pecado se ha extendido grandemente: no son solamente los varones (2,7), sino también las mujeres; andan mal quienes para proveer lujo a su vida oprimían a los pobres.

YHWH no aguantará la opresión y maltrato de pobres, y jura por su santidad que habrá castigo, que saldrán de su vida lujosa a fuerzas, llevadas con violencia. Para que esto no se cumpliera, YHWH tendría que dejar de ser santo, algo obviamente imposible. Y de hecho el cumplimiento se encuentra en 2 R 17,1-23.

En los vv. 2-3, YHWH promete un juicio a las mujeres de Israel. Con la promesa de YHWH, Amós garantiza que el juicio será realidad, porque YHWH no miente, y su santidad no puede dejar el pecado sin castigo. Advierte a un Israel

1 Este territorio es una meseta ubicada en la frontera entre Israel, Líbano, Jordania y Siria de unos $1.800 \mathrm{~km}^{2}$, de los cuales aproximadamente $1.200 \mathrm{~km}^{2}$ están ocupados por Israel, unos 235 km² están controlados por la Fuerza de las Naciones Unidas de Observación de la Separación (UNDOF) y el resto permanece bajo soberanía Siria. 
no arrepentido de su agonía venidera cuando vinieran a ser conquistados y exiliados por Asiria. Cuando los asirios despoblaron y exiliaron a una comunidad conquistada, ellos llevaron a los conquistados desnudos por viajes de cientos de millas, conectados por un sistema de hilo y anzuelos pasados por sus labios inferiores. Dios se aseguraría que fueran guiados de esta manera tan humillante por las brechas de sus ciudades conquistadas. Esto humillaría completamente a las vacas de Basán.

\subsection{Por el culto a la injusticia: vv. 4-5}

Se hace referencia, al parecer, a las fiestas sagradas celebradas en los santuarios de Israel (Betel y Guilgal). Nos referimos a los nuevos lugares para el culto instaurados por Jeroboán I, medida se tomó para evitar las peregrinaciones al Templo de Jerusalén y garantizar que los israelitas no volvieran su corazón a Jerusalén. Esto valorizó los santuarios del interior, estableciendo lugares de culto en los "lugares altos" (1R 12,31a). Esto contribuyó a descentralizar el culto, permitió el surgimiento de nuevos líderes y una mayor participación de las personas en las comunidades locales. A diferencia de Judá, en Israel los santuarios no eran dependientes de la corona, sino hasta más tarde en la historia, como veremos más adelante.

Esta medida permitió en el Reino del Norte la descentralización de la religión de la capital. Cuando David transfirió el Arca a Jerusalén (2S 6) y cuando Salomón construyó el templo (1R 7-9), lo que hacen es centralizar el culto en Jerusalén y, con esto, lograron también utilizar la religión como apoyo para el sistema monárquico. Por el contrario, Jeroboán valorizó los antiguos santuarios de los padres y de las madres de Israel, así como los de las tribus. En Betel, Abrahán había invocado el nombre de YHWH (Gn 12,8). Allí Jacob tuvo el sueño de la escalera que descendía del cielo a la tierra y edificó un altar a YHWH (Gn 28,10-22; 35,1-15). También allí las tribus consultaban a Dios (Jc 20,18; 21,2). De igual modo, Dan, en el extremo norte de Israel, fue un importante sitio de culto, en especial para la tribu de Dan (Jc 18,29-30). Betel y Dan se localizan estratégicamente en los extremos norte y sur del territorio de Israel. La actitud de Amós es irónica y despectiva al invitar a asistir a los santuarios del norte. Podría haber sido pronunciada durante la celebración de una fiesta religiosa en Betel, ante una gran muchedumbre.

Guilgal es un antiguo santuario, su nombre significa círculo (de piedras); hay varios lugares de Palestina que llevan este nombre.

En esta perícopa (vv. 4-5) vemos la ironía del profeta; lo que propone no es ir a adorar al templo como se hace en los Salmos (Sal 66,13; 100,2-4), sino que da 
una invitación sarcástica, que critica la simple ritualidad: "[o]frezcan sus sacrificios por la mañana y cada tres días sus diezmos". Algunos aducen que, en lugar de mañana y cada tres días, se debe traducir la primera mañana y el tercer día².

Cuando se habla de sacrificio, este texto intenta dar a entender que ellos ofrecieran sacrificio de paz o comunión, conocida también como sacri-

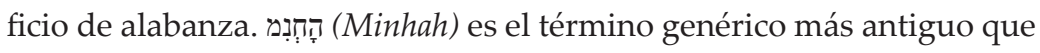
emplea la Biblia hebrea para indicar el sacrificio. Para entender este sacrificio hay que trasladarse al tiempo en el que el pueblo vivía en el desierto, cuando presentaban para sacrificio todos animales que mataban para comida de entre sus ganados, cabras u ovejas ${ }^{3}$. Este tipo de acción hizo que el pueblo creará una relación íntima con Dios. Cuando el profeta habla de los sacrificios nos acercamos a Levítico 3, donde encontramos el sacrificio de comunión. El altar de piedras del sacrificio era la mesa de Dios, Era un sacrificio con sangre, pero solamente el sebo y unas partes interiores de los animales eran quemados sobre el altar. No se quemaba toda la ofrenda; una parte era para el sacerdote y la otra la compartía quién hacia la ofrenda con su familia. Sacrificios voluntarios no eran exigidos por la ley o se hacían en cumplimiento de un voto (Ex 35, 29). El valor de sacrificios y ofrendas no depende en última instancia de la acción de las personas, sino de que $\mathrm{YHWH}$ los acepte, y en condiciones de injustica no los acepta. Pareciera que el profeta critica el interés de las personas mucho más centrado en sí mismos que en Dios, que lleva a la denuncia a la injusticia tema que se desarrolla en capítulo cinco.

2 Una posible referencia a este tema lo encontramos en $1 \mathrm{R} 12,32$, suponiendo que lo que menciona Amós aquí se refiere a que los peregrinos presentaban sus sacrificios la primera mañana de la fiesta, puede entenderse como la primera mañana después de su llegada, sus diezmos anuales son presentados el tercer día.

3 Posiblemente la diferencia cultual se deba a la institución en el Norte de un nuevo sacerdocio, es decir, fuera de las familias aceptadas en Jerusalén (1R 12,31b-32). Los sacerdotes instituidos inicialmente por Jeroboám I no eran necesariamente descendientes de Aarón, de la familia de Leví, a cuya descendencia debían pertenecer, según la tradición (Ex 4,14; 28,1). Tampoco, eran de la familia de Sadoc, cuyo linaje controlaba el poder en el templo de Jerusalén desde que Salomón confinó en Anatot a los sacerdotes del linaje de Leví (1R 2,26-27). El propio Jeroboám I se desempeñó como sacerdote (1R 12,23-33). A diferencia del Sur, en el Norte no se mantuvo el sacerdocio dependiente de la corte. En la perspectiva de los historiadores favorables al templo de Jerusalén, con toda su burocracia sacerdotal, la valorización de hombres fuera de la familia de Leví y Sadoc en Israel fue difamada y considerada un pecado. 


\subsection{Cinco Oráculos (vv. 6-11)}

Esta serie de cinco plagas o escarmientos marcados todos por la idea del castigo como una práctica saludable para el cambio, la corrección de Dios nos dirá Pablo es una misericordia (1Co 11,32). Cinco veces viene el refrán: "no habéis vuelto a mí" (vv. 6, 8, 9, 10, 11). El no arrepentimiento y la falta de conversión del pueblo serán entonces la clave de estos oráculos.

\section{a. Primer oráculo (v 6). Advertencia de YHWH}

Este oráculo nos presenta la descripción de una advertencia por parte de YHWH. Él es el causante del hambre vívida por el pueblo, pero en el fondo es el resultado de una reestructuración productiva con acumulación de tierra. Esta política va en contra del proyecto liberador que el pueblo vivió en la época tribal y que está ligada a la experiencia del Dios del Éxodo.

La experiencia de hambre en el pueblo ha sido razón de migración (Rt 1,1; Gn 12,10; 2S 21,1). Una advertencia dura por parte de YHWH quién prometió una tierra donde mana leche y miel $($ Ex 3,8$)$ es decir abundante y rica la que impide las migraciones es más es tierra donde se protege al forastero (Ex 22,20; Sal 146).

\section{b. Segundo oráculo (vv. 7-8)}

Las hambrunas, a las que hace referencia el v.6, se debían también a sequías (1 R $17,1 ; 18,2)$.

La experiencia de ausencia de la lluvia, que resulta en la sequía de los pozos, no es como la que se ve en $1 \mathrm{R} 17$ o en Jr 14; esta es caprichosa: llueve en unas ciudades y no lo hace en otras, según el texto. Podrán beber agua, pero no saciarán su sed porque siguen lejos de YHWH. El pueblo no se vuelve a YHWH. En contraposición a la afirmación del texto de no saciar nunca la sed, en Jn 4,14, Jesús ofrecerá un agua que si se bebe que hará que no se tenga más sed.

\section{c. Tercer oráculo (v. 9)}

Una nueva llamada de atención al pueblo por parte de Dios: ahora se habla de las heridas personales con la madera a medio quemar - el tizón - y lo que la sequía, la falta de agua, no ha destruido lo hará el añublo, el paracito que ataca a los cereales. Podemos ver ataques" similares en Dt 28,22 y 1 R 8,37: 
Una plaga de langostas como la que atacó a los egipcios por el endurecimiento del corazón de Faraón (Ex 10, 1-20); atacarán las huertas, los viñedos, las higueras y los olivares, es decir dejando sin nada de comer ni nada que comerciar para conseguir alimentos con los pueblos vecinos como era la costumbre (Gn 47).

\section{d. Cuarto oráculo (v. 10)}

La escala de destrucción es cada vez mayor, la descripción es aterradora: se amenaza con la misma peste que se describe en Ex 9,3-7, esto es la que atacó a Egipto con el fin de liberar al pueblo. El uso que se hace de este elemento muestra la importancia de la denuncia de injusticias que se vive. La otra imagen utilizada es la muerte de los jóvenes, lo que anula de cierta forma otra promesa de Dios: la de tener una descendencia como estrellas del cielo (Gn 26,4). No es YHWH el que anula esta promesa con la imagen de la guerra, es el pueblo que lo hace, una vez más, con la negación de volverse a YHWH, es decir cuando sigue oprimiendo a sus hermanos y de la vivencia de un individualismo y lucro que no da lugar a Dios.

\section{e. Quinto Oráculo (v. 11)}

La catástrofe de Sodoma y Gomorra, la transformación a la que el texto se refiere, pasa por el fuego, la destrucción, desaparición de lo que aleja al pueblo de Dios, el individualismo, la escalada de la injusticia, la explotación de los pobres. Algunos ejemplos de esta trasformación se pueden encontrar en Dt 29,22, Is 1,7, Jr 49,18. Pese al fuego de la destrucción, YHWH salva del fuego un pedazo de madero, que queda a medio quemar. Esto puede hacer referencia al resto de Israel $^{4}$. Los profetas utilizan y desarrollan notablemente la idea religiosa del resto. Para los profetas del período asirio, Amós, Miqueas e Isaías, el resto de Israel lo constituyen los supervivientes de las invasiones asirias de Salmanasar, Sargón y Senaquerib (Am 5,15; Is 37, 4,21; Mi 4,7).

\subsection{Sentencia Final (vv.12-13)}

Dios toma venganza de los que comenten injusticias, al igual que se ve en Is 1,24. Este dios es el dios de los ejércitos, תואבצ (Sebaot). El pueblo de Israel tiene que

4 El resto de Israel se refiere a la pequeña parte de un pueblo que se salva de la ruina decretada por Dios contra ese pueblo y que, generalmente, consiste en una invasión enemiga la primera vez que esta idea aparece es en (1R 19, 15-18). 
encontrarse con Dios. Sus prácticas religiosas (marcadas por la injusticia) y su obstinada persistencia en ellos a pesar de las llamadas al arrepentimiento han garantizado su castigo. El resultado es el ponerse a merced de su Dios, al que han ofendido. Es el dios creador, el omnipotente. Es también el dios escudriñador, el omnisciente, el dios soberano quien todas las cosas obedecen (Sal 119,91).

YHWH es un dios de justicia absoluta e inflexible. El hecho que YHWH les avisa que tendrán que encontrarse con Él es una llamada final al arrepentimiento. Saber que tenemos que aparecer ante Dios para ser juzgados es una realidad solemne: debe de hacernos reflexionar y llevarnos al arrepentimiento. En el v. 13 encontramos una de las tres doxologías presentes en el libro, es el fragmento de un himno posiblemente añadido. ${ }^{5}$ Este versículo muestra la grandeza de YHWH, Dios creador y guerrero, pero que tiene la capacidad de presentar a las personas su pensamiento de volverse a él.

\section{Conclusión}

Amós, en el capítulo cuarto, nos presenta el tema de la injusticia social desde tres perspectivas. Por un lado, está la famosa metáfora de las vacas de Basán dirigida a las mujeres de Samaría con el fin de que hablen con sus maridos. Por otro lado, se presenta el tema del culto y la conducta, relacionados nuevamente al tema

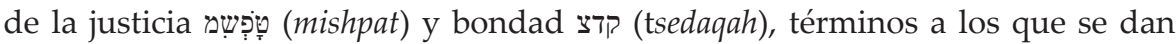
diferentes traducciones. Frecuentemente, van asociados en el Primer Testamento (PT) estos dos conceptos; siempre vienen a dar el sentido de derechos, equidad, concluyen dándonos la idea de justicia social. Por otro lado, la adoración cultual provoca el repudio de YHWH por la tergiversación que se ha dado por la injusticia social. Aquí no se ve que YHWH busque alabanza en vez de sacrificio, pues ha rechazado también los cánticos de Israel (v.5). YHWH no pide que los israelitas viajen a Jerusalén para adorarle allí ni está ordenando ninguna reforma en el culto. Demandó una sola cosa: "justicia social". Reclama justicia abundante y arrolladora como las aguas de un río y constante como un caudal que no se seca.

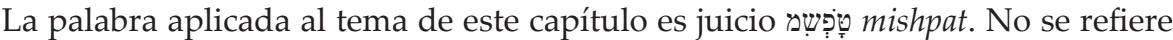
aquí al juicio divino contra el pecado, sino que es sinónimo de קדצ tsedaqah justicia. La tercera perspectiva de justicia está relacionada con el castigo de Dios, una pedagogía muy común en el PT (Gn 13,13; 19,24-25), presentada en nuestro texto

5 ASURMEDI, Jesús, Amós Amós y Oseas. Cuadernos Bíblicos 64, P. 17 
como advertencia de Dios frente a las injusticias que se están cometiendo contra los débiles y los pobres. Sin embargo, YHWH se muestra dispuesto al perdón y multiplica sus llamadas al arrepentimiento, contamos cinco solamente en este capítulo. El tono va cambiando: se acercan los nuevos tiempos, quien cumple la justicia de Dios, no se le contará la falta (Ez 18,21-24).

\section{Referencias}

\section{Referencias primarias}

Biblia de América. (1995). La casa de la Biblia (2da edición).

Biblia de Jerusalén. (1998). Bilbao: Desclée de Brouwer.

Cerni, Ricardo. (1990). Antiguo Testamento interlineal Hebreo-Español. Pentateuco T. 1. Barcelona: CLIE.

Kittel, R. (Ed). (1990). Biblia Hebraica Stuttgartensia. Stuttgart: Bibelgesellschaft.

Cantera, F., y Iglesias, M. (Ed.) (2000). Sagrada Biblia. Versión crítica sobre los textos hebreo, arameo y griego.

Rahlfs, A. (Ed.). (1979). Septuaginta Duo volumina in uno Deutsche bibelgesellschaft.

\section{Referencias secundarias}

Asurmendi, J. (2000). Amós y Oseas. Cuadernos Bíblicos, 64.

Barré, M. (2005). Amos. En R. Brown et al. (Ed.), Nuevo Comentario Bíblico San Jerónimo (pp. 320-330). Pamplona: Verbo Divino.

Cavedo, R. (1995). Profetas. Historia y teología del profetismo en Antiguo Testamento. Madrid: San Pablo.

Clarke, A. Commentary on Amos 4:1.

Recuperado de http://www.studylight.org/commentaries/acc/view.cgi?bk=29\&ch=4. 1832, 7 de febrero 2014

Domínguez García, A. (2000). Obras Completas de San Jerónimo: Comentarios a los Profetas Menores. Madrid: Biblioteca de Autores Cristianos.

Jamieson-Fausett Brown, J. (2003). Comentario exegético y explicativo de la Biblia: Antiguo Testamento. El Paso, Texas: Editorial Mundo Hispano.

Jaramillo Rivas, P. (1997). Amos. En S. Guijarro y M. Salvador, Comentario al Antiguo Testamento. Pamplona: Verbo Divino. 
Jenni, E. (1978). Diccionario teológico Manual del Antiguo Testamento. Madrid: Cristiandad.

Málek, Ludvik, et al. (2012). El mundo del Antiguo Testamento. Estella: Verbo Divino.

Menezes, R. (2005). Amos. En W. R. Farmer (Ed.), Comentario Bíblico Internacional (pp. 1030-1040). Pampolona: Verbo Divino.

Quiñónez, A., y López, J. F. (1999) Amós y Miqueas: dos profetas campesinos. Quito: Tierra Nueva - Verbo Divino.

Rostom Maderna, S. (2007). Amos. En X. Pikaza, Diccionario de la Biblia: Historia y palabra (pp. 501-513). Verbo Divino, Estella.

Schwantes, M. (1987). Amós. En, Meditaciones y Estudios. San José: Sinodal.

Storniolo, I., y Martins Balancin, E. Como leer el libro de Amós: Una denuncia de la injusticia social. Bogotá: San Pablo.

Tabet, M. (2004). Introducción al Antiguo Testamento II. Madrid: Ediciones Palabra.

Tourn, Giorgio. (1978). Amós: profeta de la justicia. Buenos Aires: Tierra Nueva.

Varo, F. (2002) Antiguo Testamento: Libros proféticos. Pamplona: Instituto Superior de Ciencias de la Religión.

Vaux, R. (1976). Instituciones del Antiguo Testamento. Barcelona: Herder.

Von Rad, G. (1990). Teología del Antiguo Testamento, vol. 2. Salamanca: Sígueme.

Westermann, C. (1987). Teologia do Antigo Testamento. São Paulo: Edições Paulinas.

Conferencia boliviana de religiosos, La lectura profética de la Historia, La Paz 1992. 